\title{
Game Analysis of the Post-Paris Agreement's Climate Policy Shao Sujun ${ }^{1, a, *}$ Rong Xue Zheng Fangyuan $^{2, \mathrm{~b}}$
}

${ }^{1}$ Yunnan University Development Institute, Kunming, Yunnan, China

${ }^{2}$ Yunnan University Development Institute, Kunming, Yunnan, China

${ }^{3}$ Yunnan University Development Institute, Kunming, Yunnan, China

a25836338@qq.com b172804658@qq.com c626127071@qq.com

* Corresponding author

Key Words: Paris Agreement; Climate Policy; Two-Level Game; Repeated Game

\begin{abstract}
The Paris Agreement is an important global agreement to cope with climate change. The withdrawal of the United States has made the implementation prospects of the Paris Agreement full of variables. This paper analyzes the post-Paris Agreement climate policy based on the two-level game theory and repeated game models. The trend of climate policy proves that the Paris Agreement will still be implemented due to its own advantages. However, if the objectives of the Paris Agreement to be fully realized, it is necessary to improve various laws and form effective constraints and incentive mechanisms.
\end{abstract}

\section{后《巴黎协议》时代气候政策的博弃分析}

\author{
邵素军 ${ }^{1, a, *}$ 戎雪 ${ }^{2, b}$ 郑芳媛 ${ }^{3, \mathrm{c}}$ \\ ${ }^{1}$ 云南大学发展研究院, 昆明, 云南, 中国 \\ 2 云南大学发展研究院, 昆明, 云南, 中国 \\ 3 云南大学发展研究院, 昆明, 云南, 中国 \\ a25836338@qq.com b172804658@qq.com c626127071@qq.com \\ 通讯作者
}

关键词：巴黎协议；气候政策；双层次博弯；重复博弯

摘要: 《巴黎协议》是全球共同应对气候变化的重要协议, 美国的退出使《巴黎协议》的执行前景充 满了变数, 本文根据双层次博弯理论和重复博弯模型, 分析了后《巴黎协议》时代全球气候政策的走 向, 证明《巴黎协议》由于自身的优势仍然会得到执行, 但是如果要充分实现《巴黎协议》的目标, 还需要完善各种制度，形成有效的约束和激励机制。

\section{1. 引言}

2017 年 6 月 1 日，美国总统特朗普宣布美国退出《巴黎协议》，此时距离《巴黎协议》生 效才刚刚半年。美国的退出给《巴黎协议》的前景蒙上了阴影，人们担心《京都议定书》的 情景又会重演。《京都议定书》美国签字了但是一直没有加入，影响了加拿大，澳大利亚，日 本等国的政策, 它们也纷纷退出, 导致《京都议定书》名存实亡, 《京都议定书》第二期有效 
期只到 2020 年，后续由《巴黎协议》来替代。

《巴黎协议》本身是人类共同应对气候变化的重大成果，不同于《京都议定书》只要求 发达国家减排和强制减排, 《巴黎协议》要求发达国家和发展中国家都参与减排并且是基于国 家自主贡献参与减排，既延续了 “共同但有区别” 的原则，又调动了全球共同参与减排的积 极性。但是美国的退出, 对后《巴黎协议》时代全球合作减排的走向增添了变数。

虽然对温室气体是否是全球变暖的元凶仍然存在争议，但是当前的共识是全球合作减排 已经关系到人类的生存和发展，特别对小岛屿国家而言更是生死存亡的事。温室气体是一种 特殊的公共产品, 它的跨国界外部效应意味着对温室气体的治理需要国家间的合作, 特别是 绝大部分国家之间的合作，这是治理温室气体的唯一出路。然而不同的国家有着不同的利益 诉求, 在合作的过程中各国通过各种方式寻求自身利益的最大化, 彼此之间进行着各种博弯, 因此博弯论是研究全球合作减排的重要方法, 而以《联合国气候变化框架公约》为基础的各 项协议的谈判, 则成了各国博弯的主要平台。

\section{2. 文献综述}

全球合作减排的政策博弯主要源于两方面，一是气候政策博弯产品的特殊性，二是气候 政策博弯主体的特殊性。气候政策博弯的产品是温室气体, 作为公共产品, 它具有非竞争性 和非排他性并具有跨国的负外部性效应。一国温室气体的排放, 不仅会影响本国, 而且也会 影响全球。同理如果一国不参与全球减排, 它也能从其他国家的减排中获益, 各国具有很强 的 “搭便车” 的动机, 导致市场失灵和政府失灵, 因此与全球合作减排不能单纯依靠市场机 制和政府政策来克服，必须通过国际合作才能有效地处理。

气候政策博弯的主体是各主权国家或国家联盟（欧盟，小岛屿国家联盟等等），不存在超 国家的机构来组织或强制各国的行动，加上各国由于不同的国情而产生不同的政治和经济诉 求，因此导致集体行动的困境，在全球合作减排中，总希望其他国家承担更多，而自己则不 仅坐享其成还能获益最多。奥尔森 (1995) 在《集体行动的逻辑》中专门探讨了集体行动的 问题，他认为出于个体理性和自利, 没有一个成员会自愿采取行动以实现集体的共同利益, 往 往选择搭便车坐享其成，因此必须完善制度的设计，引入 “选择性激励机制” 以提高成员参 与的积极性。

关于搭便车者, 阿伦 - 施密特 (1999) 认为有三类: 冒险的搭便车者、无意的搭便车者 和非自愿搭便车者。基于成本和收益的不同, 这些国家有意无意都成为了搭便车者, 不愿付 出成本，却享受着其他国家减排给自己带来的当前或长远收益。不同的国家，在全球合作减 排中搭便车的动机各不相同, 但是搭便车的动机却影响了气候政策的谈判和协议的达成与执 行（崔大鹏，2003）。

不同于普通公共产品，对于温室气体这种全球公共产品，解决搭便车问题尤其困难，究 其原因, 有以下三点: 一是减排成本高, 参与减排涉及改变产业结构、减少污染企业、发展 新能源等, 各项改革都涉及大量的资金, 影响到本国经济发展; 二是参与国家多, 参与国家 越多, 单个国家就会认为自己的贡献微不足道, 同时责任越分散, 进行国际谈判的成本也会 越高; 三是制度不尽合理, 由于缺少超越国家主权的具有强制力的国家机构, 当前的协议和 约定主要靠各国自觉遵守, 缺少外部强制约束力和内部有效激励, 导致执行困难（郡雪婷, 韦宗友, 2012)。总而言之, 搭便车的动机与全球合作减排的收益是成正比的, 收益越大, 动 机就越强, 特别是当预期成本和收益不相匹配的情况下, 就不存在主动实施减排的激励（徐 斌, 2014)。

如何解决集体行动困境, 于宏源 (2014) 认为除选择性激励措施之外有两个途径,一是有 惩罚各国的措施和规范, 使各国产生理性的预期并采取理性的经济行为, 二是通过某个霸权 
国的领导作用, 通过各种措施迫使各国参与合作减排。斯科特 - 巴雷特 (2003) 在《环境和 政府的能力》一书中也认为, 合作是解决类似气候变化这样的环境问题的唯一有效途径, 国 际社会必须调整相关机制以激励各主权国家参与全球合作减排。

针对搭便车问题, 学术界还研究了旁支付和问题链等手段。所谓旁支付, 即为了弥补某些 参与国因参与减排而遭受的损失, 其他缔约国给予的一种财政转移支付。而问题链则类似于 “以牙还牙” 策略, 将合作减排与其他合作领域（如国际贸易）相互链接起来, 促使那些不积 极参与减排的国家做出妥协。相对于旁支付, 问题链具有较强的可操作性, 是促进合作减排 和避免搭便车的有效手段。

在全球气候政策博弯分析中, 不乏用计量模型来进行分析的。刘昌新 (2013) 基于 MRICE-E 模型讨论了全球减排博弯中存在的纳什均衡。诺德豪斯 (2015) 基于 C-DICE 模型, 提出用气候 俱乐部机制来解决全球减排合作中的搭便车问题, 并证明了气候俱乐部机制的有效性。但是 大部分基于计量模型预测的结果都与现实情况严重不符, 其原因是相关模型都有严格的假设 前提, 如理性的经济人等, 同时现实的经济、政治和社会是复杂的, 模型无法穷尽所有的变 量, 因此导致了预测结果与现实相去甚远。典型的如 Juan Carlos Cícar 等（2002）基于 IAM 模型, 并融合了诺德豪斯和杨的 DICE 和 RICE 模型, 分析了后京都议定书时代气候政策, 模 型已经非常完善, 但是所得到的结果却是发展中国家比发达国家减排更加积极, 力度更大, 与事实严重的不符。

基于计量模型并不能准确地给出相关预测结果, 因此本文将抛开计量模型分析, 从双层 次博峦和无限重复序贯博峦两个理论出发, 从政治和经济的两个角度, 从空间和时间的两个 维度，对后巴黎协议时代的气候政策进行综合分析。

\section{3. 气候政策博弯分析}

无论是《京都议定书》, 还是《巴黎协议》, 全球气候协议的签订都离不开艰苦的谈判, 谈判的结果除了受到国际关系和各国的实力影响外, 也受到各国国内的政治势力和利益集团 的牵制。全球气候协议的最终签订是国内博峦和国际博弯两个层次博弯相互制衡的结果。在 国内，是各利益集团之间、利益集团与政府之间的相互博弯; 在国际层次上，是各国谈判代表 与其他国家代表之间的相互博弯，因此国际气候政策的博弯是双层次的博弯。

双层次博弯的概念最早由美国学者普特南 (1988) 提出, 该理论假设国际博弯和国内博弯 是相互交织、互动进行的, 国际层次的博弯又融合了国内层次的博弯, 国际协议的达成实际 上是基于国内博弯协议的达成。在博弯过程中，国内阶段的博弯比国际阶段的博弯更重要， 没有国内博弯的获胜, 国际博弯很难获胜。如《京都议定书》美国虽然签字了, 但是克林顿 总统没有提交国会审批，因为他没有取得国内博弯的胜利，知道不会审批通过。

获胜集合是双层次博弯理论的核心概念，是指国内选民批准国际协议所有的可能集合， 其范围大小也可以理解为国内选民批准国际协议的可能性大小。对国内选民而言, 是否批准关 键是协议对其福利的影响。不同集团获益或受损程度是不同的，二者力量的对比直接着影响 着国内博弯的结果，也决定着最终的获胜集合。

普特南认为国际协议的谈判者是没有个人独立偏好的国内利益的忠实代言人，但实际上 谈判者是有个人偏好与利益诉求的。他首先有个人利益的诉求（追求连任或者更大的声誉）, 其次他还要代表所在集团的利益诉求, 最后还要代表所在国家人民的诉求。因此谈判代表是 一个有自己利益偏好和政策主张的主体, 直接参与国内博弯, 而且国内博弯比国际博弯更重 要, 对于谈判者来说, 国内博弯的成败直接影响着他的政治生涯, 因此各国谈判代表必须同 时兼顾国际国内两方面的利益诉求。 
从国际层面看，当前国际气候政策谈判主要有以下三大集团：欧盟、以美国为首的伞形 集团和以中国为首的发展中大国。不同的集团有着不同的利益打算, 谈判的背后还掺杂着各 国国内政治力量和利益集团的相互较量。

美国为首的伞形集团包括美国、加拿大、日本、澳大利亚、新西兰等国。美国在伞形集 团扮演着领导者的角色, 影响伞形集团其他各国对气候问题国际合作的态度。美国伯德法案明 确规定总统不应签署 “对美国经济导致严重损害的” 条约。这也是《京都议定书》不能在美 国批准通过的原因, 尽管克林顿在协议中加入了碳排放交易, 但是还是未能获得国内的获胜 集合。到了奥巴马政府时期, 国内的利益团体的力量对比发生了变化, 支持新能源和绿色环 保的力量占了上风, 因此奥巴马获得国内获胜集合, 推动了《巴黎协议》在美国的批准通过。 同理特朗普上台之后, 美国国内传统能源势力又重新占据上风, 因此特朗普宣布美国退出《巴 黎协议》。所有国际决策的背后其实是各自所代表的国内利益集团之间博峦的结果。

由此可以预见, 在特朗普总统执政期间, 美国不会重新加入《巴黎协议》, 但是在碳减排 方面, 美国为了争取全球主导权, 会尝试重新构建以其为首的新的气候谈判框架。美国总统 特朗普宣布退出《巴黎协议》后, 有 211 个美国城市市长集体承诺仍将接受《巴黎协议》, 这 说明美国国内的博弯仍在继续, 并影响着美国的国际政策。不管怎样, 由于《巴黎协议》自 身的特点不同于《京都议定书》, 因此美国虽然退出了《巴黎协议》, 但是伞形集团的其他国 家预计不会退出《巴黎协议》, 因为参与协议远比退出协议所获得的收益更大。

基于生态利益、经济利益和政治利益的追求，欧盟一直是全球合作减排的积极倡导者。

首先, 基于生态利益的诉求。全球气候变化对欧洲的影响日益凸显, 欧洲近年来经常遭遇 极端气候, 同时欧洲很多国家海拔普遍偏低, 全球变暖将危及很多临海国家的生存。随着经 济的发展, 欧洲更加关注对环境福利的追求, 在某种意义上, 环保主义已经成为欧洲民族主义 的一个组成部分。

其次, 新能源技术的推广, 将为欧盟创造巨大的经济利益。当前欧盟在新能源技术、碳排 放机制、节能与环保标准、低碳项目推广等各方面, 均走在世界各国的前列。全球合作减排将 给欧洲的新能源技术创造巨大的利润空间。

最后, 通过推动全球合作减排将给欧盟带来新的政治收益。与美国消极态度相比, 欧盟一 直是积极的全球合作减排推动者, 并且以身作则推动减排, 欧盟的积极表现为其赢得了很好 的国际声誉，提升了其在国际政治中的影响力，成为国际气候合作机制的制定者。

虽然受欧债危机的影响, 部分欧盟国家经济发展疲弱, 再加上英国脱欧, 削弱了欧盟的 整体实力, 但是气候环境的恶化将直接威胁到欧盟公民正在享有的环境福利, 这使得欧盟的 谈判者和国内选民有着共同的利益交集, 具有很大的获胜集合。而美国的退出为欧盟提升其 国际政治影响力提供了极好的机会, 同时欧盟内部在气候政策方面保持着一致, 没有伞形集 团各国之间存在的分歧, 因此预测欧盟会严格遵守《巴黎协议》, 并积极开展各种国际合作, 最大化其环境利益、经济利益和政治利益。

以中国为首的发展中国家, 特别是金砖五国, 随着经济的发展, 成为温室气体的排放大 户, 也成为了国际气候谈判中发达国家针对的主要目标。因此为了更好地参与国际合作, 并提 升发展中国家的影响力, 以中国为首的发展中国家积极推动《巴黎协议》的谈判与签约, 没 有发展中国家的努力, 特别是中国的努力, 《巴黎协议》是无法达成并生效的。随着发展中国 家在国际政治经济影响力的日益提升, 以及《巴黎协议》的减排是基于国家的自主贡献, 美 国的退出不会触及各国国内集团的利益，因此预计发展中国家不会退出《巴黎协议》。

《巴黎协议》基于国家自主贡献的减排机制，有效地解决了全球减排义务分配的难题， 实现了国内博弯和国际博弯的均衡, 获得了最大的获胜集合, 因此基于双层次博弯分析, 美 
国退出《巴黎协议》不会影响《巴黎协议》的执行。

上述分析只能得出《巴黎协议》仍将得到执行，但是执行效果如何，能否完全实现《巴 黎协议》的目标, 还需要从时间维度的重复博弯进行分析。从时间来看, 国际气候政策博弯 是不断重复的博弯。不同于一次博弯, 重复博弯可以避免各国基于机会主义而选择搭便车, 它可以增进参与各国的信任, 从而走出囚徒困境。

国际气候政策博弯从理论上讲是一个无限重复序贯博弯的过程, 因为只要人类生存, 只 要温室气体排放仍然存在, 各国之间始终将存在博弯。同时博弯的信息是完备的, 因为一国 是否履约, 其它国家完全可以观察到, 按照无限重复序贯博弯, 由于任何一国都有以牙还牙 报复的机会, 就会导致各国不敢违约, 因此参与者可以达到实现纳什均衡的合作。但是实际 中由于参与国际气候政策谈判代表的政治任期是有限的, 而且谈判代表有个人偏好和利益诉 求, 在这种情况下, 博弯对他而言就不是无限次数的, 而是有限次数的, 在有限次重复博弯 中, 囚徒困境博弯的纳什均衡是参与者的不合作。当然如果谈判代表的国内利益与国际利益 是重合的，即合作可以得到最大的获胜集合，则仍然可以实现合作的纳什均衡。

因此《巴黎协议》虽然仍然得到执行，但是执行效果如何，仍然取决于各国之间的博弯。 为了避免搭便车以及各国谈判者单纯追求个人的政治利益, 国际气候政策的谈判仍然需要加 强组织与制度建设, 探索各种机制包括有效的激励与约束机制, 避免因个别国家不履约而导 致的多米诺骨牌效应。

\section{4. 气候政策博恋的模型分析}

上述从理论方面进行了分析，本部分将从模型方面进行分析。国际气候政策博弯的主体 很多，但是主要的博弯还是发生在发达国家和发展中国家之间。

图 1 描述了发达国家与发展中国家之间的博弯，博弯的两个参与者：发达国家集团和发 展中国家集团, 两个集团都面临三种策略选择, 即合作 (C), 表示合作减排; 不作为 $(\mathrm{N})$, 表示原有排放量保持不变; 恶化 (W), 表示将进一步增加排放量。博弯涉及的成本和收益分 别表示如下: $\mathrm{R}$ 表示合作产生的公共收益, $\mathrm{c}$ 表示行动各国各自承担的成本, $\mathrm{C}$ 表示恶化温 室气体排放带来的公共成本, $r$ 表示恶化给该国带来的单独收益。假设 $2 \mathrm{R}>\mathrm{c}>\mathrm{R}$, 且 $2 \mathrm{C}$ $>r>C$, 这表示合作给两个集团带来的总收益大于各自付出的成本，单独行动则会遭受净损 失; 而单方面增加排放量会获益，但两个集团同时增加排放量则会出现公地悲剧。

\section{发展中国家}

\begin{tabular}{|c|c|c|c|c|}
\hline & & $\mathrm{C}$ & $\mathrm{N}$ & W \\
\hline \multirow{3}{*}{ 发达国家 } & $\mathrm{C}$ & 2R-c,2R-c & $\mathrm{R}-\mathrm{c}, \mathrm{R}$ & $\mathrm{R}-\mathrm{c}-\mathrm{C}, \mathrm{R}+\mathrm{r}-\mathrm{C}$ \\
\hline & $\mathrm{N}$ & $\mathrm{R}, \mathrm{R}-\mathrm{c}$ & 0,0 & $-\mathrm{C}, \mathrm{r}-\mathrm{C}$ \\
\hline & W & $\mathrm{R}+\mathrm{r}-\mathrm{C}, \mathrm{R}-\mathrm{c}-\mathrm{C}$ & $\mathrm{r}-\mathrm{C},-\mathrm{C}$ & $\mathrm{r}-2 \mathrm{C}, \mathrm{r}-2 \mathrm{C}$ \\
\hline
\end{tabular}

图 1 发达国家与发展中国家的博弯

根据上文的分析, 该博弯的纳什均衡是不合作，各国都选择恶化 (W, W), 对应的博弯收 益为 $(r-2 C, r-2 C)$ 。当然图 1 表示发达国家与发展中国家同时行动, 实际执行当中并不如此, 因为发达国家和发展中国家对温室气体的容忍程度是不同的。在环境与发展的权衡中, 发展 中国家比发达国家更倾向于经济发展。于是，在发展中国家与发达国家的减排博栾中，发展 中国家比发达国家更有耐心，发达国家比发展中国家更承受不起环境破坏所带来的损失，因 
此发达国家将率先采取减排行动 $(\mathrm{C})$ 。对发展中国家而言则仍然存在三种选择, $\mathrm{C}, \mathrm{N}$ 和 $\mathrm{W}$, 不 同策略的收益是 $R+r-C>R>2 R-c$, 即恶化是最好的选择。发达国家为了让发展中国家参与行动, 必须对发展中国家提供额外的收益 $e$, 使 $2 R-c+e>R+r-C$, 或者提出惩罚措施 $f$, 使 $R+r-C-f<2 R-c$, 或者是激励与惩罚相结合, 来使发展中国家选择 $\mathrm{A}$ 。但是当发达国家选择激励或惩罚的时候 会影响国内集团的利益, 因此首先要进行国内博弯, 然后再进行国际博弯, 同时是激励还是 惩罚, 发达国家之间需要保持一致, 这又涉及到发达国家之间的博弯。图 2 以博弯树的方式 对此进行了补充，描述了国家集团间重复序贯博弯的情形。

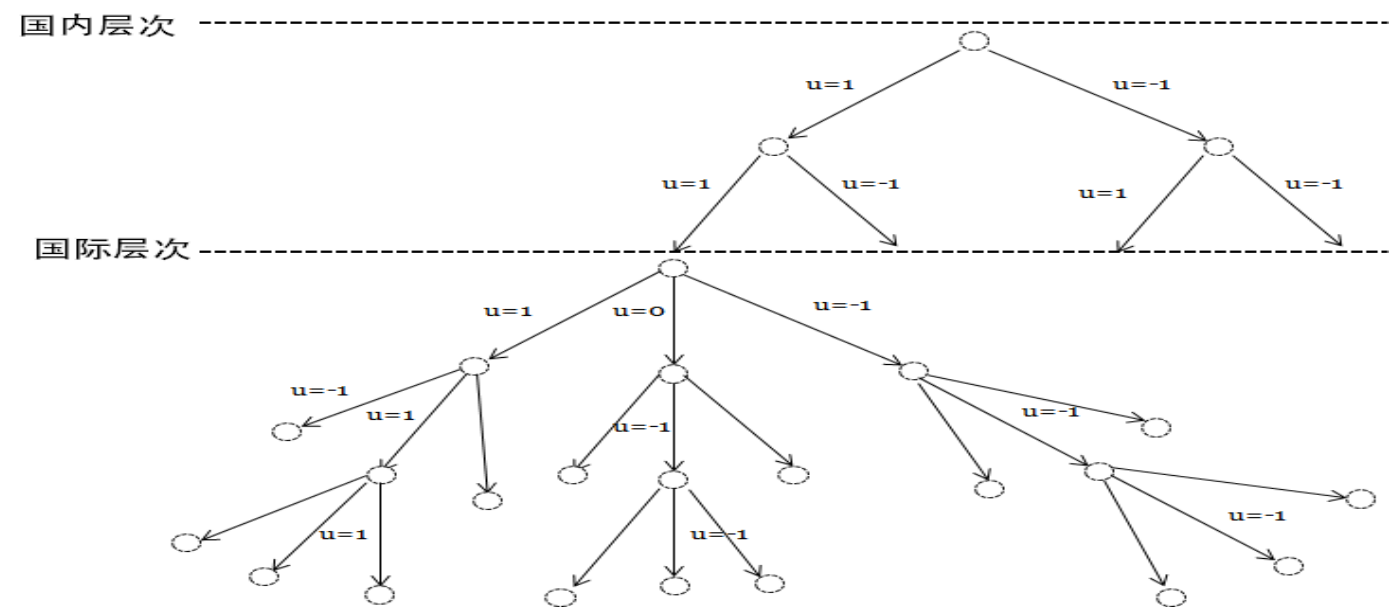

图 2 发达国家与发展中国家双层次重复序贯博弯

从国家层面来看, 因为涉及国内各利益集团之间的博弯, 所以决策的结果只有两个, 一 个是参加 $(u=1)$, 一个是不参加 $(u=-1)$, 而且只有获得国内同意之后才能参与国际层面的博 弯, 但是在国际层面, 一国为了获得最大效益, 可以选择三个策略, 一是合作 $(u=1)$, 二是 搭便车 $(u=0)$, 三是恶化 $(u=-1)$ 。但由于博弯不是一次性博弯, 而是序贯重复博弯, 因此其 它国家有以牙还牙的策略, 即如果采取了搭便车或者恶化策略, 则其它国家会采取同样的措 施予以回击, 因此只有合作才是均衡。但是在这个过程中不能排除个别国家谈判代表由于政 治任期及政治目的而采取搭便车或者恶化的策略, 以获得个人利益的最大化, 因此合作的均 衡是不稳定的, 存在一定的不确定性。

通过上述分析，双层次重复序贯博弯存在着合作的纳什均衡的可能，即让发展中国家和 发达国家在合作下的收益大于不作为或者恶化下的收益。如何实现, 制度和机制的设置非常 重要。诺德豪斯 (2015) 提出的成立气候俱乐部并对非成员进行相应惩罚, 可以说就是基于 上述思想。但是他的博恋模型基于两大假设前提：一是所有参与成员符合理性人假设; 二是 一次博弯与重复博峦的结果完全相同。而这两种假设与现实都不相符, 因此基于模型的预测 是不可能实现的。

不仅发达国家之间存在博弯，发展中国家之间也存在着博弯。在气候政策博弯中，发展 中国家也有三种策略可供寻选择, 一是恶化 (W), 即增加温室气体排放, 二是不作为 (N), 即保持原有排放量不变, 三是有策略行动 ( T ) , 即以获取最大的国际收益和国内收益为目标, 但不一定真正履约减排。此时成本和收益的关系变为 $2 \mathrm{C}>\mathrm{r}>\mathrm{C}$ 和 $\mathrm{c}>\mathrm{R}>0$ 。 
发展中国家 2

\begin{tabular}{|c|c|c|c|c|}
\hline & & W & $\mathrm{N}$ & $\mathrm{T}$ \\
\hline \multirow{3}{*}{ 发展中国家 1} & W & $r-2 C, r-2 C$ & $\mathrm{r}-\mathrm{C},-\mathrm{C}$ & $\mathrm{r}-\mathrm{C}, \mathrm{R}-\mathrm{C}-\mathrm{c}$ \\
\hline & $\mathrm{N}$ & $-\mathrm{C}, \mathrm{r}-\mathrm{C}$ & 0,0 & $\mathrm{R}, \mathrm{R}-\mathrm{c}$ \\
\hline & $\mathrm{T}$ & $\mathrm{R}-\mathrm{C}-\mathrm{c}, \mathrm{r}-\mathrm{C}$ & $\mathrm{R}-\mathrm{c}, \mathrm{R}$ & $\mathrm{R}-\mathrm{c}, \mathrm{R}-\mathrm{c}$ \\
\hline
\end{tabular}

图 3 发展中国家之间的博弯

由于 $r>C$ 且 $R>0$ ，因此不作为（N）是严格的劣策略，该策略可以被剔除，图形可以简 化为图 4 , 参与国只有 $\mathrm{W}$ 和 $\mathrm{T}$ 两个策略可选择。在这种情况下, 则需要权衡 $\mathrm{R}+\mathrm{C}$ 和 $\mathrm{r}+\mathrm{c}$ 之间 的大小, 如果 $R+C>r+c$, 则有策略行动 ( $T$ ) 就是占优策略, 如果 $R+C<r+c$, 则恶化 (W) 就是 该博弯的占优策略。

\section{发展中国家 2}

\begin{tabular}{|c|c|c|}
\hline & W & $\mathrm{T}$ \\
\hline \multirow{2}{*}{ 发展中国家 1} & $r-2 C, r-2 C$ & $\mathrm{r}-\mathrm{C}, \mathrm{R}-\mathrm{C}-\mathrm{c}$ \\
\hline & $\mathrm{R}-\mathrm{C}-\mathrm{c}, \mathrm{r}-\mathrm{C}$ & $\mathrm{R}-\mathrm{c}, \mathrm{R}-\mathrm{c}$ \\
\hline
\end{tabular}

图 4 发展中国家之间的简化博弯

由于发展中国家数量庞大, 不同的国家基于不同的国情有着不同的追求目标, 在最大化 本国利益的过程中容易与其它国家发生冲突, 因此博峦的结果更加充满不确定性。比如石油 输出国组织因为担心全球温室气体减排将影响本国的石油销量和价格, 一直是采取消极的合 作态度。

\section{5. 结束语}

基于上述理论分析和模型分析，后《巴黎协议》时代，《巴黎协议》将继续得到执行，但 是执行效果仍然取决于参与各国的国内博弯和国际博弯。由于温室气体排放公共产品的特殊 性和参与气候政策谈判国家主体的复杂性，后《巴黎协议》时代的气候政策的执行效果存在 着不确定性。

如何有效实现博弯的纳什均衡, 如同上文所分析的, 关键是让参与国的收益大于成本, 或者是违约的成本大于收益。因此可以尝试基于《巴黎协议》制定相关的机制, 其中一个可 行的机制是碳排放权交易机制。《京都议定书》有效期到 2020 年, 京都议定书执行过程中, 发达国家如欧盟、日本、澳大利亚、新西兰等都建立了碳排放权交易体系, 美国虽然没有加 入《京都议定书》, 但它有自发的碳排放权交易体系, 2017 年中国作为最大的发展中国家也 在全国范围内启动了碳排放权交易体系。目前各个碳排放交易体系基本是割裂的, 但是如果 将已经启动碳排放交易体系国家的碳排放交易体系融合在一起，统一进行碳排放权的交易， 类似于诺德豪斯的气候俱乐部, 而且由于该气候俱乐部既包含了发达国家又包含了发展中国 家, 再辅之以问题链的处理方法, 即对不能遵守《巴黎协议》的国家, 通过国际贸易如征收 碳关税的方式予以惩处, 则可以在全球范围内促使《巴黎协议》的履行与预期目标的实现。

另外，《京都议定书》确立的清洁发展机制对《巴黎协议》的执行仍然有很强的借鉴价值， 各国在合作减排的过程中关注的是各个国家的可持续发展, 通过该机制, 发达国家可以实现 
相关技术的商业化，而发展中国家则可以获取相关的技术和资金，这是一种可以实现双赢的 机制。有鉴于《京都议定书》相关机制的重要性，因此《巴黎协议》在签署的前言中仍然强 调 “迫切需要加快执行《公约》及其《京都议定书》，以加强 2020 年之前的力度”。

因此基于《京都议定书》碳排放权交易形成气候俱乐部，基于清洁发展机制形成发达国 家与发展中国家的合作, 再辅之以问题链的处理方法, 各国之间的博弯可以实现稳态的纳什 均衡，因此可以实现《巴黎协议》的预期目标。

\section{References}

[1] Matthew Kennedy, Biswajit Basu, An analysis of the climate change architecture, Renewable and Sustainable Energy Reviews 34 (2014) 185-193

[2] S.C. van Pelt, Marjolijn Haasnoot, Communicating climate (change) uncertainties: Simulation games as boundary objects, environmental science \& policy 45 (2015) 41-52

[3] T. Balint, F. Lamperti, Complexity and the Economics of Climate Change: A Survey and a Look Forward, Ecological Economics 138 (2017) 252-265

[4] Stephen J. DeCanio, Anders Fremstad, Game theory and climate diplomacy, Ecological Economics 85 (2013) 177-187

[5] Gao Lei, Chen Junhua, Shen Changcheng, Research on climate diplomacy based on game theory[J]. Journal of Southwest China Normal University (Natural Science Edition), 2015-06.

[6] Lou Lingli, The Essence of Environmental Cooperation under the Framework of "two-level game theory" - Taking Multilateral Climate Change Negotiations as An Example [J], World Economics and Political Forum, 2008, 04.

[7] Wang Fangjun, Zhang Shiguo, National Climate Protection Strategy Analysis Based on Reputation Game[J], Journal of Chongqing University of Technology (Social Sciences), 2017(01).

[8] Wei Mei, Research on Global Carbon Emission Reduction Cooperation and Development of Climate Game Experiment Platform under the Climate Club Mechanism[D]. Master thesis of East China Normal University, 2016.

[9] Liang Jiao, Research on Game Analysis and Mechanism of Low-carbon Economy International Cooperation[D], Master thesis of Southwest Jiaotong University, 2011. 